\title{
Contrast Performance: Low Voltage Electrons vs. Helium Ions
}

\author{
David C. Bell
}

\author{
School of Engineering and Applied Sciences, and Center for Nanoscale Systems, \\ Harvard University, Cambridge, Massachusetts 02138
}

In order to achieve ultra high resolution imaging with secondary electron imaging, it is critical that the electric potential of the specimen surface is well controlled. For electrically conductive samples this can be achieved by simply grounding the specimen. However, imaging of electrically insulating specimens can provide challenging or impossible to image due to uncompensated charge resulting from the electron or ion beam interaction with the specimen surface. The main reason for the uncompensated charge is that the insulating specimen has insufficient conductivity through mobility of either electrons or holes to quickly restore the neutrality of the scanned area (Fig. 1.) The buildup charge causes significant deflection and distortion of the ion or electron beam. Which is more appropriate, to use charge compensation with high $\mathrm{kV}$ helium Ions or employ a low $\mathrm{kV}$ SEM image to obtain the required surface information? This paper will present a systematic examination of the surface information provided by both techniques, including SEM charge compensation mechanisms.

Charge compensation in SEM on insulating samples can be achieved via various techniques: a) employing specimen bias to effectively 'drain' some of the charge; b) flood gun; and c) ultra-low kV imaging in sub $0.5 \mathrm{kV}$ regime. Another approach is to employ different image acquisition techniques, for example line integration or frame averaging to enable image acquisition without beam deflection; though this approach often fails and produces images with inadequate $\mathrm{S} / \mathrm{N}$ ratio. Moreover, even with these approaches, there are still specimens and observation conditions (for instance very high tilt) that are difficult to image in the SEM. One key advantage of the Helium Ion Microscope technology in the case of imaging highly charging specimens is the electron flood gun can be utilized to neutralize the positive charge buildup and facilitate high-resolution imaging (Fig. 2.). A flood gun is used to charge the surface to a negative potential (using electrons as the neutralizing particles). When utilizing the electron flood gun, the electron beam and He ion beam are synchronized and adjusted with respect to one another, so that the low energy electrons are not interfering with the secondary electron imaging.

Some of our research from the past year has been surprising and may provide a foundation for a change in analysis techniques of different materials. The imaging and contrast mechanisms are different in comparison to standard SEM imaging and provide further details of materials and especially insulating materials and other compositions that have been previously unattainable or at the least difficult to acquire [1]. The nature of the Helium ion beam interactions with the sample shows enhanced edge contrast which is especially useful for critical dimension measurements; one particularly interesting development is the imaging of non-conducting materials showing a contrast due to three apparent mechanisms simultaneously atomic number, channeling contrast and a possible enhanced edge contrast (Fig. 2). The advantages of Helium ion microscopy is still being investigated (while the conventional SEM has been improving for decades), there are still many more discoveries waiting.

\section{References}

[1] D.C. Bell "Contrast Mechanisms and Image Formation in Helium Ion Microscopy", Microscopy and Microanalysis, 15 (2009), pp. 147-153 

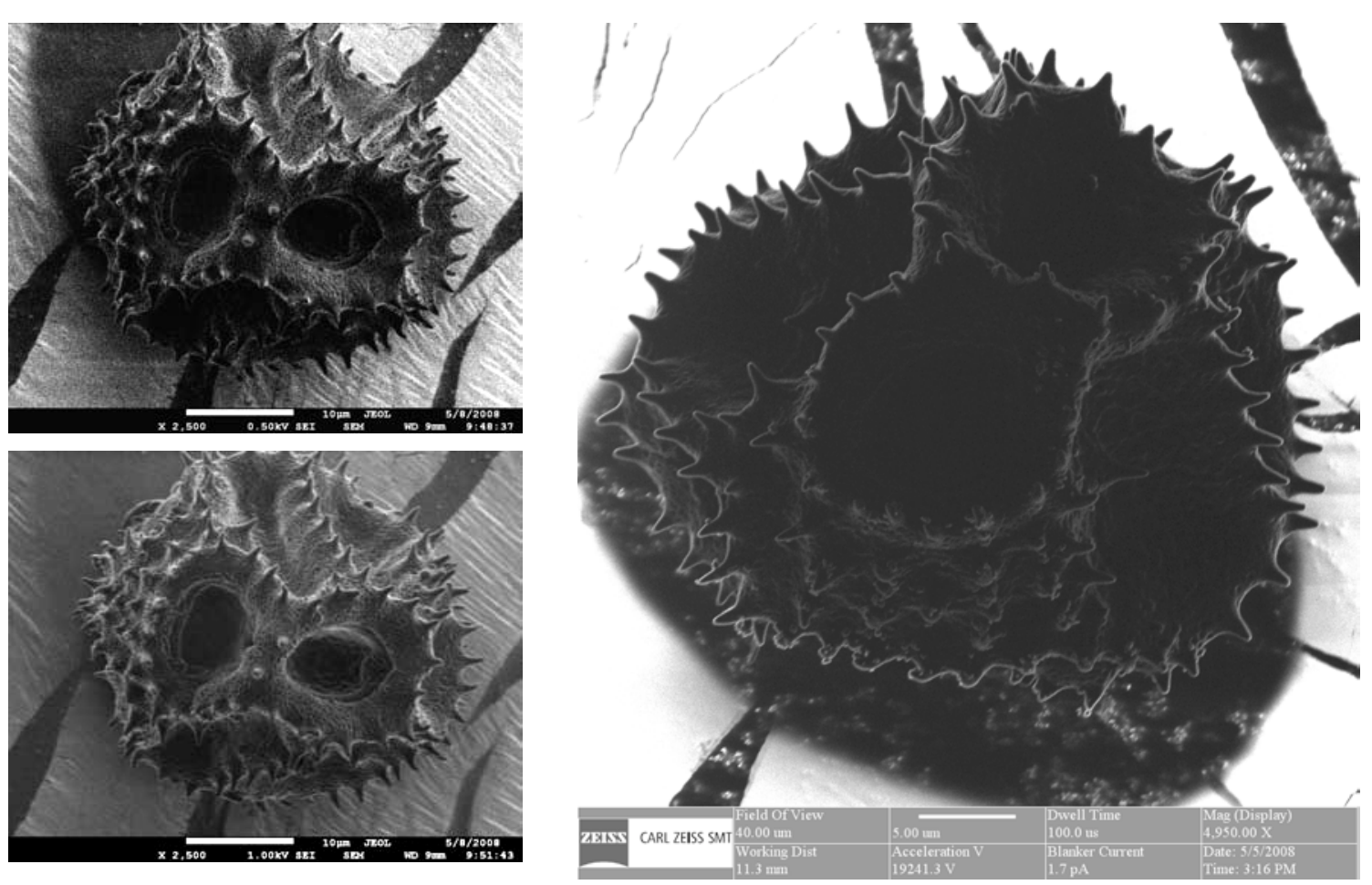

Figure 1. Example: images of pollen (uncoated). Left - SEM images showing reversal of contrast between $0.5 \mathrm{kV}$ image (top) and $1 \mathrm{kV}$ image (bottom). Right - HeIM image of the same sample, showing the similarity to a low $\mathrm{kV}$ SEM image.
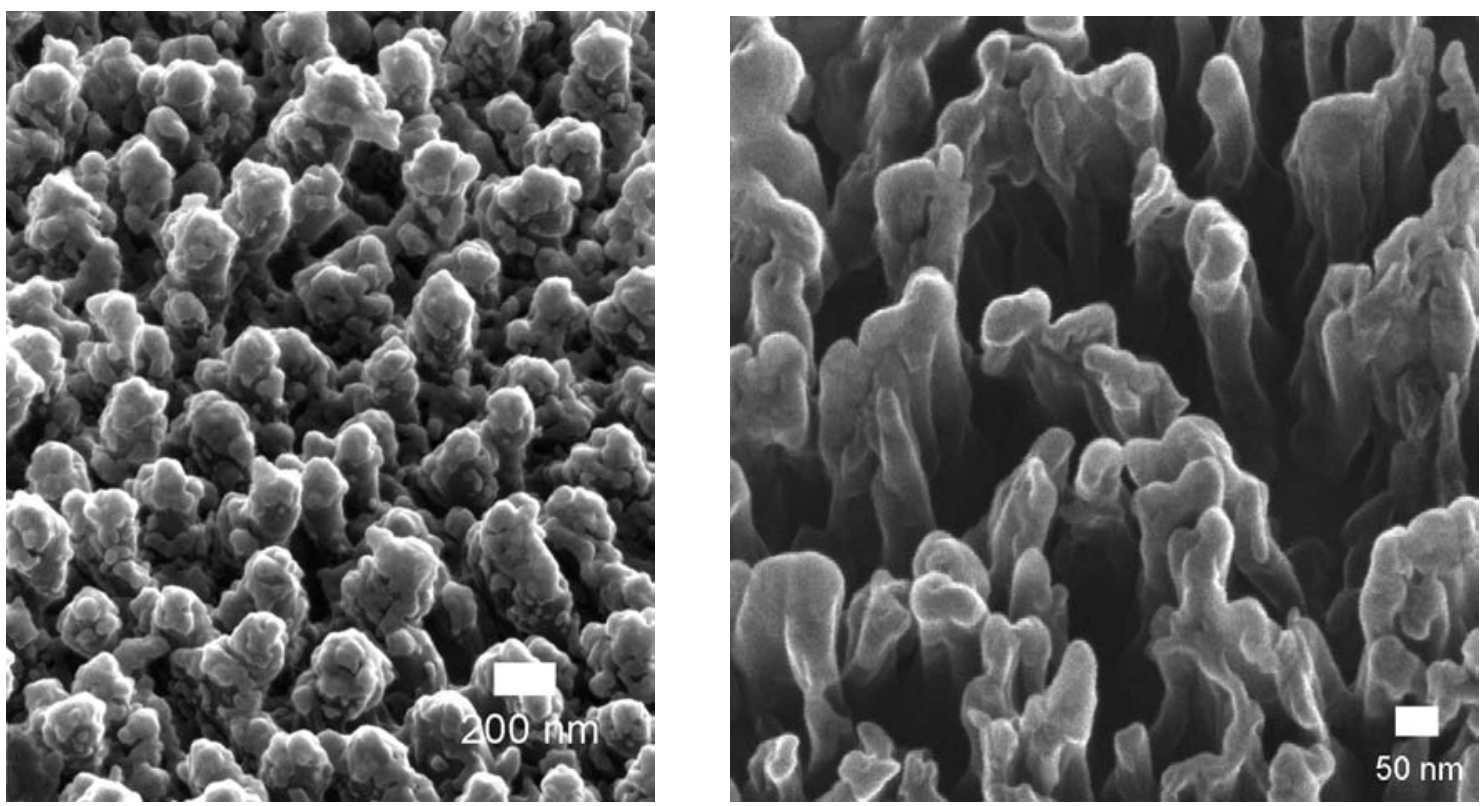

Figure 2. Left, High-Resolution, Helium Ion Microscope image of LSLN SERS substrate. Note that the silver forms aggregates of particles on the surface, as opposed to a continuous film. Right, Helium Ion Microscope image of a black etched silicon film with high surface area, that is extremely difficult to image in the SEM. 